\title{
Scanning electrochemical microscopy studies for the characterization of localized corrosion reactions at cut edges of coil coated steel
}

\author{
B.M. Fernández-Pérez ${ }^{1}$, J. Izquierdo ${ }^{1,2}$, S. González ${ }^{1}$, R.M. Souto ${ }^{1,2, *}$ \\ ${ }^{1}$ Department of Chemistry, University of La Laguna, 38071 La Laguna, Tenerife, Spain. \\ ${ }^{2}$ Instituto Universitario de Materiales y Nanotecnologías, University of La Laguna, E-38200 La \\ Laguna (Tenerife, Canary Islands), Spain \\ *E-mail: rsouto@ull.es
}

\begin{abstract}
The local distribution of anodic and cathodic sites in a corroding cut edge using the scanning electrochemical microscope is presented. Platinum and antimony microdisks were used as tips for combined amperometric/potentiometric operation. Local changes in electrochemical activity, oxygen concentration and $\mathrm{pH}$ distribution associated to the onset of localized anodes and cathodes were imaged in situ, as well as their evolution with time. Local alkalization and oxygen consumption related to cathodic activity were detected above the zinc layer covered with the thinner polymeric coating, providing a direct evidence of a differential aeration process effectively operating in an asymmetrical cut edge, in addition to the galvanic coupling of aluzinc and steel. Anodic activity leading to local acidification occurred at the other aluzinc layer at all exposures.
\end{abstract}

Keywords: SECM; galvanic corrosion; concentration distributions; cut edges; aluzinc; steel. 


\section{Introduction}

Polymer coated zinc and zinc alloy galvanized steels are widely employed in building and transport industries due to the convenient combination of good mechanical properties of the steel, whereas gaining effective anticorrosion resistance by means of the thin galvanized layers and the barrier organic coating. Yet corrosion protection often fails at these systems due to small defects present in the coating, such as holes or flaws produced during either the manufacturing or the handling of the material, and most especially from cut edges. The big difference in dimensions between the steel matrix and the metallic and polymeric layers applied for its protection also limits the amount of metal available for sacrificial protection. Therefore, defects and cut edges in the micrometric range are a major issue determining the adequate performance of these materials. This is the reason that localized techniques are explored to study the degradation reactions at polymer-coated galvanized steels, though only the scanning Kelvin probe (SKP) [1-4] and the scanning vibrating electrode technique (SVET) [5-7] have contributed to characterize these systems from cut edges. Unfortunately, these techniques lack chemical resolution, and the species involved contribute to these signals despite their chemical identity.

The application of scanning electrochemical microscopy in conventional amperometric mode has facilitated an advance in the understanding of corrosion reactions at polymer coated galvanized steels, by enabling the highly localized early stages of the process to be analyzed [8$11]$, and even monitor the ability of novel coating systems to promote self-healing to defective systems $[12,13]$. But amperometric microdisks exhibit serious limitations for the quantification of some species of interest, mainly metal cations with sufficiently negative redox potentials for which the faradaic current associated with their reduction cannot be separated from the total current in the system [14,15]. Additionally, local pH changes accompany most corrosion processes in aqueous environment, yet they pass unnoticed using amperometric SECM. Though local $\mathrm{pH}$ evolution can be monitored by SECM in potentiometric operation since 1993 using either an antimony microdisk [16] or an ion selective microelectrode as tip [17], this procedure has not been applied in Corrosion Science until very recently $[18,19]$. In this way, evidence of local cathodic activity on the more active metal within a model zinc-iron galvanic couple could be detected $[18,19]$. More interestingly, since combined amperometric/potentiometric operation can be achieved in SECM, the quasi-simultaneous analysis of most of reactions involved in the corrosion phenomena has become feasible [20-22]. This experimental approach is very powerful in the case of complex microstructural systems. It is well-known that the grain size reduction increases the metal tensile strength, such as the yield strength is proportional to the reciprocal of the square root of the grain diameter [23-26]. Yet, regarding the corrosion behaviour, it is not always true that a finer microstructural array induces an improvement in the corrosion resistance [24-25]. Indeed, coarse grains may exhibit a better corrosion behaviour than fine ones. It has also been reported that coarse equiaxed grains of hot-dip galvanized samples tend to improve 
the corrosion resistance when compared with fine equiaxed grains [24-25]. This is associated with the energy level at the grain boundaries due to plastic deformation and the occurrence of defects [24-26].

In organic coated and galvanized metal systems, the chemical interactions between the various materials are of major interest. They involve the results of eventual physical damages, the establishment of galvanic couples, and the formation of corrosion products. Consequently, chemical phenomena occurring at cut edges are complex processes in which the different interfaces interact with the environment in the micrometer scale. In this work, a cut edge system consisting on organic coating / galvanization layer / mild steel material was investigated in natural $\mathrm{pH}$ solution, and both amperometric and potentiometric scans were measured using SECM. Data obtained in each mode show good correlation and allow characterization of the processes involved.

\section{Experimental}

\subsection{Materials}

A commercial painted galvanized carbon steel system was considered for the investigation of cut edge processes. The thickness of the steel foil was $400 \mu \mathrm{m}$ while the galvanised layer was a $20 \mu \mathrm{m}$ thick metallic aluzinc coating (nominal composition of $55 \%$ zinc and $45 \%$ aluminium). Next, a chromate layer was applied prior to painting with the polymeric coating. A polyester paint system consisting of $5 \mu \mathrm{m}$ thick primer containing strontium chromate as inhibitor was applied to either side of the metallic sheets, whereas a single $20 \mu \mathrm{m}$ thick topcoat containing $\mathrm{TiO}_{2}$ was applied only to one side. Samples of size of $20 \mathrm{~mm}$ by $20 \mathrm{~mm}$ were cut from rolled pannels with a guillotine from the topcoat side downwards. The cut edge was embedded in an Epofix resin sleeve (Struers, Ballerup, Denmark) that only their cross sections were exposed on the front side of the disc-shaped resin mounting (dia. $3 \mathrm{~cm}$ ). Cut edges of approximately $20 \mathrm{~mm}$ length and $450 \mu \mathrm{m}$ width were produced. Figure 1 depicts a top-view micrograph and a sketch of the cut edge. The cut edge surface was ground to mirror surface with silicon carbide paper down to 4000 grit, followed by polishing with $0.3 \mu \mathrm{m}$ particle size alumina in ethanolic suspension. The specimens were subsequently washed with $96 \%$ ethanol in an ultrasonic bath, and dried in air. A container for approximately $3.5 \mathrm{~mL}$ of test solution was created surrounding the mount laterally by Sellotape. In this way, the cut edge was exposed upwards to the test solution, the side with the primer and the topcoat was placed at the right side in order to facilitate comparison between different experiments. Tests were conducted in stagnant $1 \mathrm{mM}$ (millimols per litre) $\mathrm{NaCl}$ solution, naturally aerated, and at ambient temperature $\left(20 \pm 2{ }^{\circ} \mathrm{C}\right)$. 


\subsection{Scanning electrochemical microscopy}

\subsubsection{Instrument}

A SECM equipment supplied by Sensolytics (Bochum, Germany) was employed for spatially-resolved characterization of the electrochemical reactivity of the cut edge specimens exposed to an aqueous electrolyte. The instrument was built around an Autolab bipotentiostat provided with a frequency response analyzer, all controlled with a personal computer. The samples were tested at their open circuit potentials in the test solutions. The electrochemical cell was completed with an $\mathrm{Ag} / \mathrm{AgCl} /(3 \mathrm{M}) \mathrm{KCl}$ electrode as reference $\left(E^{O}=+0.197 \mathrm{~V}\right.$ vs. $\left.\mathrm{NHE}\right)$, and a Pt plate of as counter electrode (approximate area: $0.72 \mathrm{~cm}^{2}$ ). All potential values are referred to the $\mathrm{Ag} / \mathrm{AgCl} /(3 \mathrm{M}) \mathrm{KCl}$ electrode. The same SECM instrument was employed for the potentiometric measurements using $\mathrm{pH}$-sensitive tips, though a home made voltage follower based on a $10^{12} \Omega$ input impedance operational amplifier had to be interconnected between the cell and the potentiometric input of the system [27]. Line scans were made using a step/acquire scheme where the step size was $10 \mu \mathrm{m}$, whereas grid images were acquired by moving the microelectrode in a raster-type motion. The scan rate was $7 \mu \mathrm{m} / \mathrm{s}$, and the tip-substrate height was set at $15 \mu \mathrm{m}$. SECM data were plotted using either Microcal Origin or Quickgrid softwares for the scan lines and the 2D images, respectively. Experiments were performed in triplicate to assure reproducibility of the measurements, and typical measurements are given without application of any fitting or filtering procedures.

\subsubsection{Amperometric operation}

A platinum microdisc of diameter $10 \mu \mathrm{m}$ was employed as the SECM tip. Operation in this mode implies the addition of a reversible or quasi-reversible redox couple to the electrolyte. When this redox substance, the electrochemical mediator, is added in its reduced state and the ultramicroelectrode (UME) is biased to the potential of oxidation of the mediator under diffusion conditions, a limiting current, $i_{\text {lim }}$, is defined by eqn. (1), for a disc shaped UME:

$$
i_{\lim }=4 n F D c a
$$

where $F$, is the Faraday constant; $a$, the microelectrode radius; $D$, the diffusion coefficient of the reducible species; $n$, the number of electrons exchanged; and, $c$, its concentration. That is, a steady-state current results from the constant flux of $O$ to the electrode surface due to an expanding hemispherical diffusion layer around the microelectrode. Ferrocene-methanol of concentration $0.5 \mathrm{mM}$ was added to the test solutions to act as electrochemical mediator at the tip. This chemical species was chosen because the redox potential for its reversible oxidation to ferrocinium ion, according equation (2), occurs at potential values very positive (+0.5 V vs. $\mathrm{Ag} / \mathrm{AgCl} /(3 \mathrm{M}) \mathrm{KCl})$ to the open circuit potential of the cut edge $(-0.8 \mathrm{~V} \mathrm{vs}$. $\mathrm{Ag} / \mathrm{AgCl} /(3 \mathrm{M}) \mathrm{KCl})$. 
Thus, ferrocene-methanol should be the only stable species from this redox couple present in the electrolyte, and it should not react directly or cause any potential modifications on the substrate. SECM data were drawn using the dimensionless current $\left(I=i / i_{\text {lim }}\right)$, where $i_{\text {lim }}$ is the limiting current when the tip was far away from the surface. Positioning of the tip in relation to the specimen was established by recording the $z$-approach curves over the resin at both sides of the cut edge, thus allowing for any tilt of the sample to be compensated.

\subsubsection{Potentiometric operation}

Electrodes made of antimony and coated by the metal oxide have the ability to behave as a $\mathrm{pH}$ sensor. Using a gas flame, melted antimony from metal powder is introduced in a Pyrex glass capillary under suction. Antimony-filled fibers are then fabricated using a pipette puller system, and they are introduced in the lumen of a micropipette with the tip reaching out for about $15 \mathrm{~mm}$. Electric contact is ensured by inserting liquid mercury metal and a copper wire in the lumen of the micropipette. Loctite ${ }^{\circledR}$ adhesive was used to seal the two micropipette ends. Discshaped tips are thus obtained, with an active antimony electrode at the centre, surrounded by glass. The dimensions of the active metal surface were determined by cyclic voltammetry in a deoxygenated $\left[\mathrm{Ru}\left(\mathrm{NH}_{3}\right)_{6}\right] \mathrm{Cl}_{3}$ solution, showing a reduction peak at around $-0.2 \mathrm{~V}$ vs. $\mathrm{Ag} / \mathrm{AgCl} / \mathrm{KCl}(3 \mathrm{M})$. The antimony microelectrode had a $40 \mu \mathrm{m}$ diameter active disc surface.

Next, the antimony electrode was polarized positive to its open circuit potential to produce an oxide layer. In order to determine localized $\mathrm{pH}$ distributions in the solution side next to the cut edge, the antimony electrode was calibrated from the measurement of the potential response transients toward $\mathrm{pH}$ change of the solution, using a sequence of eight buffer solutions covering the $4 \leq \mathrm{pH} \leq 11$ range. The calibration procedure was initiated with the most alkaline solution, and the tip was sequentially exposed to solutions of increased acidity as shown in Figure 2A. The overpotential values observed in the plot occurred upon electrolyte exchange, and next the electrode attained a stead potential value in each solution. Finally, the electrode was reintroduced in the most alkaline buffer solution to check the reproducibility of its potential response. With the potential values taken from each buffer solution, the calibration plot depicted in Figure 2B was drawn. The system shows a good linear relationship, with slope $-48.2 \mathrm{mV} / \mathrm{pH}$ unit.

Positioning of the antimony tip close to the surface was assisted using a video camera. The tip was allowed to gently rest on the sample, and subsequently the probe was retracted to the chosen operation distance (namely $15 \mu \mathrm{m}$ ) with the aid of the $z$-positioning motor. 


\section{Results}

When the Pt tip is brought to the vicinity of an insulating substrate, the steady-state current that flows through the tip, $i$, tends to be smaller than $i_{\text {lim. }}$. This is a result of the insulating substrate partially blocking the diffusion of $O$ towards the tip. The current at the tip becomes smaller when the tip is closer to the substrate, and tends to zero when the distance between tip and substrate, $d$, approaches zero. This effect is known as negative feedback. Normalized currents smaller than 1 are observed. Conversely, if the tip is close to a conductive substrate at which the oxidation reaction (3) can occur, a flux of $O$ from the substrate to the tip occurs, in addition to some flux from the bulk solution towards the tip.

$$
\mathrm{FCMeOH}^{+}+\mathrm{e}^{-} \rightleftarrows \mathrm{FCMeOH}
$$

This effect leads to an enhancement of the current at the tip, $i$, which is higher than $i_{\text {lim. }}$ Then the flux of $\mathrm{FcMeOH}$ from the substrate to the tip causes an increase of $i$ as $d$ decreases. In this case, when the tip-substrate distance approaches zero, the $i$ current tends to infinite, and the oxidation of $\mathrm{FcMeOH}$ on the substrate is diffusion-controlled. This effect is named positive feedback. The tip can be seen as both the generator of the signal sensing the substrate (the flux of the oxidized species, $\mathrm{FcMeOH}^{+}$), and the detector (of the flux of $\mathrm{FcMeOH}$ from the substrate).

For the tip travelling over the cut edge at constant height, negative feedback behaviour should occur when placed above the epoxy sleeve, whereas positive feedback will occur over the metal, as schematically drawn in Figure 3. The corroding metal substrate provides the electrons required for the regeneration of the reduced formed of the redox mediator, and this happens though the cut edge sample is not externally polarized in the experiments [22]. This issue is very relevant because the system is allowed to corrode spontaneously in the environment while the SECM measurements can be conducted in situ.

Figure 4A shows a selection of scan lines recorded over an arbitrary line taken across the cut edge. These curves were measured after different durations of the exposure of the system to $1 \mathrm{mM} \mathrm{NaCl}$ solution. The location of the metallic components of the cut edge become readily observable from both scan lines and 3D images, as indicated by those regions leading to normalized currents exceeding one at the tip due to the regeneration of the redox mediator due to the availability of electrons in the substrate even at its open circuit potential. Though both the steel foil and the aluzinc layers contributed to the positive feedback effect, higher feedback values occurred on steel than on the aluzinc layers (cf. Figure 4A). The time evolution of these scan lines indicates that progressive blocking of the metallic surface occurs with the elapse of time in this test electrolyte, resulting in a smaller difference between the faradaic currents measured at the tip while passing over the steel compared to the background levels maintained over the insulating resin. The distribution of the chemical reactivity along the length of the cut 
edge can be imaged by scanning a 2D grid extending along both $x$ and $y$ axes, as shown in Figure 4B. This SECM map was recorded after 4 hours exposure of the sample to the test solution, i.e. immediately after the series of scan lines given in Figure $4 \mathrm{~A}$ were acquired. It is demonstrated that these scan lines, which were taken through the cut edge in order to follow the time evolution of its electrochemical activity, are representative of the situation along the whole cut edge.

The onset of the cathodic reaction could also be followed by setting the potential of the platinum tip at $-0.65 \mathrm{~V}$ vs. $\mathrm{Ag} / \mathrm{AgCl} /(3 \mathrm{M}) \mathrm{KCl}$. At this potential, reduction of oxygen naturally dissolved in the electrolyte occurs at the platinum microelectrode according to:

$$
\mathrm{O}_{2}+4 \mathrm{e}^{-}+2 \mathrm{H}_{2} \mathrm{O} \rightarrow 4 \mathrm{OH}^{-}
$$

This reaction is not reversible and therefore cannot be investigated in the feedback mode. In this case, tip and substrate are competing for the same reaction because oxygen is a reactant of the cathodic reaction occurring at the sample (equation (4)). A decrease in the reduction current at the tip will then correspond to oxygen depletion at the probe, normally associated with a cathodic region. Therefore, stationary tip currents are expected to be measured when the tip travels over the insulating sleeve around the cut edge, whereas a depletion of oxygen concentration in the electrolyte solution volume adjacent to the cathodic sites in the corroding system would lead to a competition between the tip and the substrate for the reduction of this molecule. As a result, smaller current values should be measured at the tip when it passes over the steel substrate, because the metal is expected to be the cathode when galvanically coupled to the aluzinc layers, as schematically shown in Figure 5. In this way, the different electrochemical activity of the steel plate and the aluzinc layers in the corrosion process is better observed from the selected scan lines taken at various times along a line crossing the cut edge shown in Figure 6. Due to the consumption of this molecule at the cathodic sites in the cut edge, variations were observed in the oxygen reduction current monitored at the tip. The current decreased locally to values close to zero at early exposures. Additionally, at the shortest times, oxygen consumption mainly occurred over a portion of the steel plate, and progressively covered all the steel. Asymmetrical consumption of oxygen on the two aluzinc layers can also be observed, with greater consumption of oxygen occurring on the aluzinc/steel interface placed at the left. Only at the longest exposures, oxygen got almost completely depleted over all the cut edge.

Changes in the solution $\mathrm{pH}$ close to the substrate, which are associated with the corrosion process, could be monitored when the antimony microelectrode was employed for the potentiometric operation of the SECM. In naturally-aerated aqueous solutions, the cathodic reaction is the reduction of dissolved oxygen in the electrolyte according to equation (4), which leads to alkalization of the electrolyte close to the cathodic sites. Due to the galvanic coupling between the aluzinc and the steel in the cut edge, the aluzinc surface becomes less alkaline, 
because the cathodic activity would occur on the steel strip. Furthermore, zinc dissolution occurs in competition with metal hydrolysis as described by equation (5), thus contributing to additional acidification next to the anodic sites.

$$
\mathrm{Zn}^{2+}+m \mathrm{H}_{2} \mathrm{O} \rightleftarrows \mathrm{Zn}(\mathrm{OH})_{m}^{2-m}+m \mathrm{H}^{+}
$$

The combined effects of reactions (4) and (5) are shown schematically in Figure 7. Though not indicated in the graph for the sake of simplicity, the occurrence of aluminium dissolution in competition with metal hydrolysis must also be considered to occur (cf. equation (6)).

$$
\mathrm{Al}^{3+}+n \mathrm{H}_{2} \mathrm{O} \rightleftarrows \mathrm{Al}(\mathrm{OH})_{n}^{2-n}+n \mathrm{H}^{+}
$$

Co-dissolution and hydrolysis of Al along with $\mathrm{Zn}$, since aluzinc contains $45 \%$ of aluminium, would produce even lower $\mathrm{pH}$ values in the electrolyte volumes adjacent to the anodic regions developed in the cut edge [28].

Heterogeneous distribution of $\mathrm{pH}$ over the cut edge was observed for exposures up to $132 \mathrm{~min}$, whereas the $\mathrm{pH}$ started to homogenize in the scan line measured after 224 min (see Figure $8 \mathrm{~A}$ ). For the latter, blocking of the cut edge surface due to the precipitation of corrosion products occurred. Indeed, the localization of corroding anodes and cathodes was not homogeneously distributed in the material, as depicted by the $3 \mathrm{D}$ image given in Figure $8 \mathrm{~B}$ extending over all the cut edge.

\section{Discussion}

Combined amperometric/potentiometric operation of the SECM supply information on the distribution of anodic and cathodic regions developed on a cut edge of painted zinc alloy galvanized steel during exposure to an aqueous electrolyte solution containing aggressive chloride ions. Metal dissolution at the anodic sites, and oxygen consumption at the cathodic ones, are thus monitored together with the local $\mathrm{pH}$ changes produced in the electrolyte next to those reacting regions. Furthermore, the changes with time of these processes can be followed too, thus delivering valuable experimental information on the nature and evolution of the reactions involved.

A major difficulty faced in this investigation was related to the level of lateral resolution that can be provided by the scanning electrochemical technique for a better description of the corroding system. Surface roughness and the combination of the dimensions of the surface region to scan and those of the smaller features to be detected, are major issues as to establish the lateral resolution. In our investigation, since the cut edge was embedded in an insulating epoxy resin sleeve, and the exposed surface was subjected to a sequence of abrading and polishing steps, it can be regarded that a sufficiently flat surface was imaged. This statement is 
not rigorously true, because the different hardness of the cut edge components and the resin sleeve will lead greater removal of material from the soft materials than from the metal parts during abrading and subsequent polishing. Yet, the differences can be regarded to amount less than a couple of microns, thus allowing us to consider that all the responses measured at the scanning tip originated exclusively from local variations in electrochemical reactivity in the system. Secondly, the major difference in relative sizes of the steel plate and the aluzinc galvanized layers must also be considered. The cross section taken through the cut edge will extend $400 \mu \mathrm{m}$ above the steel plate, and only $25 \mu \mathrm{m}$ over each aluzinc layer. As a compromise between the length of the scan required to cross over the whole cut edge thickness and adequately resolve the different materials, platinum microdisc electrodes of $10 \mu \mathrm{m}$ diameter where regarded to allow the aluzinc layers to be resolved to at least 2-3 points each. Bigger tips would not resolve those metallic coatings, whereas even smaller tips, though eventually providing greater lateral resolution for the aluzinc layers, will require longer times to travel over the whole cut edge. The latter would greatly compromise time resolution of the process, because the system could undergo significant changes during the acquisition of one single line scan. The same applies to the recording of 2D maps of the complete cut edge. Therefore, time evolution of the process was followed by recording a series of scan lines over the same portion of the cut edge, whereas complete 2D images were only measured at selected times when minor changes were observed between subsequent scan lines. Finally, it should be indicated that the instrument set up allowed for the scanning conditions to be modified between consecutive scan lines. In this way, consecutive lines could be recorded in the different operation modes of the SECM, the different parameters were measured almost at the same time. More strictly, they were actually monitored just after completing the previous scan line for another parameter.

Electrochemical activity related to galvanic corrosion coupling between the steel body and the protecting aluzinc layers applied either side of the plate is observed during exposure of a cut edge to $1 \mathrm{mM} \mathrm{NaCl}$ solution. The activation of the system, and the eventual partial blocking of the corroding surface at longer exposures as indicated by the measurement of smaller faradaic currents at the platinum tip travelling over the cut edge is thus evidenced from the inspection of Figure 4. They are related to metal dissolution from the aluzinc layers at either side. This blocking effect must be due to the precipitation of corrosion products on the cut edge. Though the precipitation of zinc oxides could not be supported by thermodynamic calculations in near neutral bulk solutions, recent reports using scanning electron micrographs in combination with energy-dispersive analysis have shown the appearance of nodules of zinc oxides directly formed from the surface of zinc [29], and within defects in coated zinc alloy galvanized steel [30].

The cathodic half cell reaction is also visualized from the measurement of the local depletion of dissolved oxygen concentration occurring around the cathodic sites developed on the cut edge. As expected, oxygen consumption is observed when the platinum tip passes over 
the steel plate, whereas the amount of oxygen available over the aluzinc layers is bigger at least at the beginning of the experiment. Yet the sequence of scan lines given in Figure 6 clearly indicated that some additional oxygen consumption occurs over the aluzinc layer situated at the left (i.e., the metallic coating covered with the thinner polyester film). This differential consumption of oxygen over the two aluzinc layers is an observation that the anodic process occurs at the aluzinc layer coated by the thickest organic layer. That is, differential aeration corrosion due to restricted access of oxygen to the aluzinc layer proximal to the thicker organic coating occurred [31].

This differential aeration corrosion mechanism is further demonstrated by inspecting the $\mathrm{pH}$ distribution data given in Figure 8. Alkalization occurred above the steel strip and the aluzinc layer at the left, whereas acidification happened only at the right of the cut edge due to the hydrolysis of the metal ions released in the anodic oxidation of zinc:

$$
\begin{aligned}
& \mathrm{Zn} \rightarrow \mathrm{Zn}^{2+}+2 \mathrm{e}^{-} \\
& \mathrm{Zn}^{2+}+\mathrm{H}_{2} \mathrm{O} \rightarrow \mathrm{Zn}(\mathrm{OH})^{+}+\mathrm{H}^{+}
\end{aligned}
$$

and aluminium:

$$
\begin{aligned}
& \mathrm{Al} \rightarrow \mathrm{Al}^{3+}+3 \mathrm{e}^{-} \\
& \mathrm{Al} l^{3+}+\mathrm{H}_{2} \mathrm{O} \rightarrow \mathrm{Al}(\mathrm{OH})^{2+}+\mathrm{H}^{+}
\end{aligned}
$$

Although additional hydrolysis of $\mathrm{Al}(\mathrm{OH})^{2+}$ to $\mathrm{Al}(\mathrm{OH})_{2}{ }^{+}$and further could be possible, it is unlikely to occur in the naturally corroding environment due to the accumulation of $\mathrm{H}^{+}$from the first stage of the hydrolysis reaction. The sequence of reactions given in equations (7)-(10) leads to acidification around the anodic sites [28]. Though zinc could be expected only to produce some weak acidification due to its rather high hydrolysis constant $\left(p K_{1, Z n}=8.0\right)$, aluminium dissolving along with zinc would originate further acidification because its corresponding hydrolysis constant $\left(p K_{1, A l}=4.5\right)$ is smaller [32].

The reported asymmetrical microelectrochemical data related to differential aeration at either side of the sample due different thicknesses of the painted layers applied on the coil coating steel has been observed for the first time using an embedded sample. Though restricted access of oxygen to the sacrificial metallic coating through the thicker coating has been well established for free-standing cut edges (i.e., non embedded) [31, 33, 34], the process could not be investigated with the scanning electrochemical microscope until now due to lack of lateral resolution. The fixation of the cut edge using a resin matrix of a different nature than that used in the painted layers (respectively epoxy and polyester), may originate different rates for oxygen and effectively lead to the similarity of the differential aeration in the real system as compared to the embedded system. Though this explanation may be regarded to be speculative at this stage, new studies are currently designed at our laboratory with the goal to test the validity of this 
assumption by employing model assemblies with more controlled geometries and compositions for the cut edge system.

\section{Conclusion}

Localized distributions of both anodic and cathodic activity on an aluzinc galvanized cut edge have been imaged in situ using SECM in combined amperometric/potentiometric operation. This method allowed the detection of different reactivities at each aluzinc layer originating from the different thicknesses of the two polymer coating films applied on them. Furthermore, changes in electrochemical activity over the cut edge with the elapse of time were monitored, whereas imaging the distribution of different species participating in the process with high lateral resolution. Changes on the oxygen concentration and the $\mathrm{pH}$ in the solution related to cathodic activity were observed.

A differential aeration mechanism is invoked to explain the differences in reactivity observed between the two aluzinc layers applied either side of the mild steel plate, which originate from they being coated with polymeric paint films of different thickness at each side.

\section{Acknowledgments:}

This work was supported by the Spanish Ministry of Economy and Competitiveness, and the European Regional Development Fund (Project No. CTQ2009-14322). A Grant awarded to J.I. by the Spanish Ministry of Education (Programa de Formación de Personal Investigador) is gratefully acknowledged. The Authors acknowledge the valuable comments raised by the reviewers.

\section{References}

1. Williams G, McMurray HN, Worsley DA (2002) Cerium(III) inhibition of corrosion-driven organic coating delamination studied using a scanning Kelvin probe technique. J Electrochem Soc 149:B154-B162

2. Paliwoda-Porebska G, Stratmann M, Rohwerder M, Potje-Kamloth K, Lu Y, Pich AZ, Adler $\mathrm{H}-\mathrm{J}$ (2005) On the development of polypyrrole coatings with self-healing properties for iron corrosion protection. Corros Sci 47:3216-3233

3. Whyte EP, Sykes JM Behaviour of a zinc-iron bimetallic couple coated with poly-vinyl butyral lacquer during intermittent exposure to salt solution. Corros Sci 49 (2007) 3361-3380 
4. Muto I, Sato K, Hara N (2010) Scanning Kelvin probe analysis of cut edge corrosion on prepainted galvanized steel with chromate-containing epoxy primer. ECS Transactions 2529:59-70

5. Ogle K, Baudu V, Garrigues L, Philippe X (2000) Localized electrochemical methods applied to cut edge corrosion. J Electrochem Soc 147:3654-3660

6. Ogle K, Morel S, Jacquet D (2006) Observation of self-healing functions on the cut edge of galvanized steel using SVET and pH microscopy. J Electrochem Soc 153:B1-B5

7. Alvarez-Pampliega A, Lamaka SV, Taryba MG, Madari M, De Strycker J, Tourwé E, Ferreira MGS, Terryn H (2012) Cut-edge corrosion study on painted aluminum rich metallic coated steel by scanning vibrating electrode and micro-potentiometric techniques. Electrochim Acta 61:107-117

8. Souto RM, González-García Y, González S (2005) In situ monitoring of electroactive species by using the scanning electrochemical microscope. Application to the investigation of degradation processes at defective coated metals. Corros Sci 47:3312-3323

9. Simoes AM, Battocchi D, Tallman DE, Bierwagen GP (2007) SVET and SECM imaging of cathodic protection of aluminium by a Mg-rich coating. Corros Sci 49:3838-3849

10. Walsh DA, Li LE, Bakare MS, Voisey KT (2009) Visualisation of the local electrochemical activity of thermal sprayed anti-corrosion coatings using scanning electrochemical microscopy. Electrochim Acta 54:4647-4654

11. Santana JJ, González-Guzmán J, Fernández-Mérida L, González S, Souto RM (2010) Visualization of local degradation processes in coated metals by means of scanning electrochemical microscopy in the redox competition mode. Electrochim Acta 55:4488-4494

12. González-García Y, Mol JMC, Muselle T, De Graeve I, Van Assche G, Scheltjens G, Van Mele B, Terryn H (2011) SECM study of defect repair in self-healing polymer coatings on metals. Electrochem Commun 13:169-173

13. Pilbáth A, Szabó T, Telegdi J, Nyikos L (2012) SECM study of steel corrosion under scratched microencapsulated epoxy resin. Prog Org Coat 75:480-485

14. Souto RM, González-García Y, Battistel D, Daniele S (2012) In situ SECM detection of metal dissolution during zinc corrosion by means of mercury sphere-cap microelectrode tips. Chem Eur J 12:230-236

15. Souto RM, González-García Y, Battistel D, Daniele S (2012) On the use of mercury-coated tips in scanning electrochemical microscopy to investigate galvanic corrosion processes involving zinc and iron. Corros Sci 55:401-406

16. Horrocks B, Mirkin MV, Pierce DT, Bard AJ, Nagy G, Tóth K (1993) Scanning electrochemical microscopy. 19. Ion-selective potentiometric microscopy. Anal Chem 65:1213-1224

17. Denuault G, Nagy G, Nagy L (2012) Potentiometric probes. In: Bard AJ, Mirkin MV (eds) 
Scanning electrochemical microscopy, 2nd edition. CRC Press, Boca Raton, pp. 275-315

18. Izquierdo J, Nagy L, Varga Á, Santana JJ, Nagy G, Souto RM (2011) Spatially resolved measurement of electrochemical activity and $\mathrm{pH}$ distributions in corrosion processes by scanning electrochemical microscopy using antimony tips. Electrochim Acta 56:8846-8850

19. Izquierdo J, Nagy L, Varga Á, Bitter I, Nagy G, Souto RM (2012) Scanning electrochemical microscopy for the investigation of corrosion processes: measurement of $\mathrm{Zn}^{2+}$ spatial distribution with ion selective microelectrodes. Electrochim Acta 59:398-403

20. Izquierdo J, Nagy L, Santana JJ, Nagy G, Souto RM (2011) A novel microelectrochemical strategy for the study of corrosion inhibitors employing the scanning vibrating electrode technique and dual potentiometric/amperometric operation in scanning electrochemical microscopy: Application to the study of the cathodic inhibition by benzotriazole of the galvanic corrosion of copper coupled to iron. Electrochim Acta 58:707-716

21. Souto RM, Kiss A, Izquierdo J, Nagy L, Bitter I, Nagy G (2013) Spatially-resolved imaging of concentration distributions on corroding magnesium-based materials exposed to aqueous environments by SECM. Electrochem Commun 26:25-28

22. Izquierdo J, Nagy L, González S, Santana JJ, Nagy G, Souto RM (2013) Resolution of the apparent experimental discrepancies observed between SVET and SECM for the characterization of galvanic corrosion reactions. Electrochem Commun 27:50-53

23. Petch NJ (1953) The cleavage strength of polycrystals. J Iron Steel Inst 174:25-31

24. Osório WR, Freire CM, Garcia A (2005) The role of macrostructural morphology and grain size on the corrosion resistance of $\mathrm{Zn}$ and Al castings. Mater Sci Eng A 402:22-32

25. Osório WR, Siqueira CA, Freire CM, Garcia A (2005) Effects of the longitudinal and transversal structural grain morphologies upon the corrosion resistance of zinc and aluminium specimens. Rev Metal Madrid 41SI:176-180

26. Osório WR, Cheung N, Spinelli JE, Garcia A (2007) The effects of a eutectic modifier on microstructure and surface corrosion behavior of Al-Si hypoeutectic alloys. J Solid State Electrochem 11:1421-1427

27. Izquierdo J, Nagy L, Bitter I, Souto RM, Nagy G (2013) Potentiometric scanning electrochemical microscopy for the local characterization of the electrochemical behaviour of magnesium-based materials. Electrochim Acta 87:283-293

28. Lamaka SV, Souto RM, Ferreira MGS (2010) In situ visualization of local corrosion by scanning ion-selective electrode technique (SIET). In: Méndez-Vilas A, Díaz J (eds) Microscopy: Science, Technology, Applications and Education, Vol. 3. Formatex Research Center, Badajoz, pp. 2162-2173

29. Thomas S, Birbilis N, Venkatraman MS, Cole IS (2013) Corrosion of zinc as a function of pH. Corrosion 68:015009-1-9

30. Souto RM, Normand B, Takenouti H, Keddam M (2010) Self-healing processes in coil coated 
cladding studied by the scanning vibrating electrode. Electrochim. Acta 55:4551-4557

31. Bohm S, McMurray HN, Powell SM, Worsley DA (2000) Photoelectrochemical investigation of corrosion using scanning electrochemical techniques. Electrochim Acta 45:2165-2174

32. Lur'e YY (1989) Handbook of analytical chemistry, $6^{\text {th }}$ edition. Himiya, Moscow

33. Worsley DA, McMurray HN, Belghazi A (1997) Determination of localised corrosion mechanisms using a scanning vibrating reference electrode technique. Chem Commun 2369-2370

34. Worsley DA, Williams D, Ling JSG (2001) Mechanistic changes in cut-edge corrosion induced by variation of organic coating porosity. Corros Sci 43:2335-2348
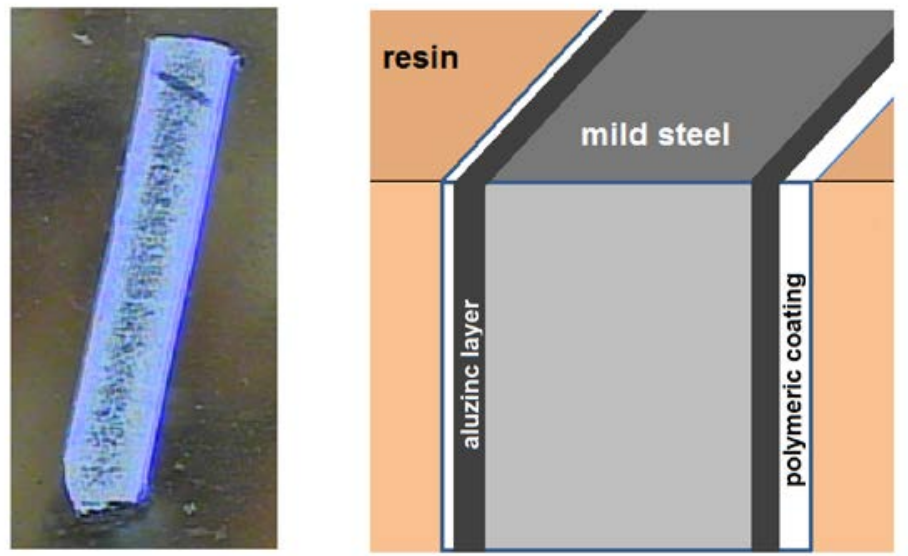

Figure 1 - (A) Top-view micrograph and (B) sketch of the cut edge.
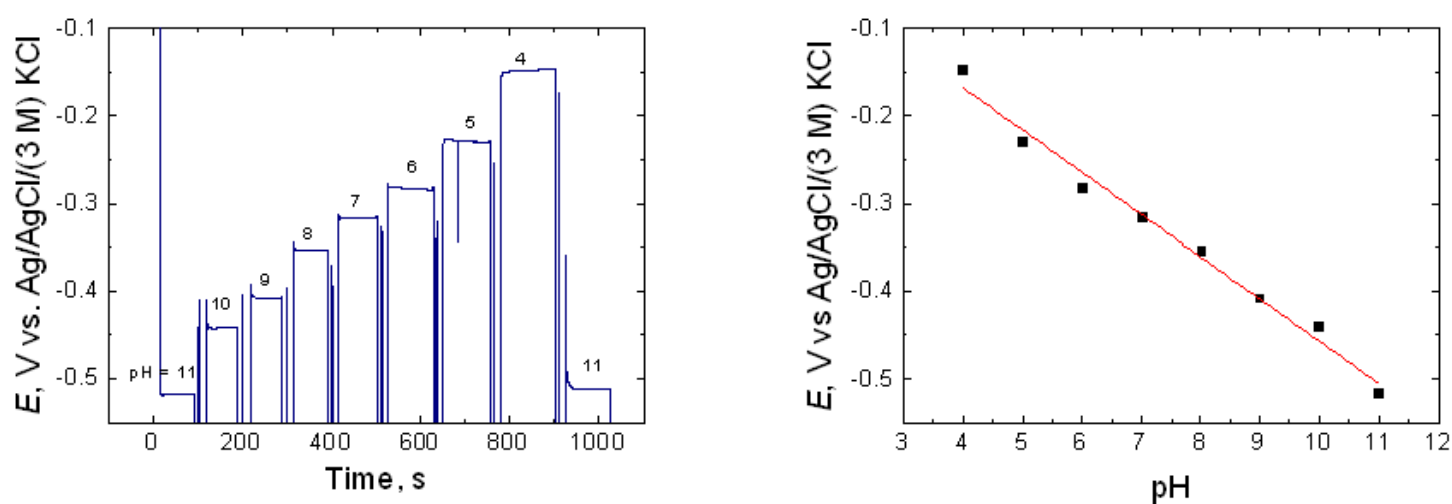

Figure 2 - (A) Dynamic response transients of the antimony microelectrode to $\mathrm{pH}$, and (B) calibration plot. 


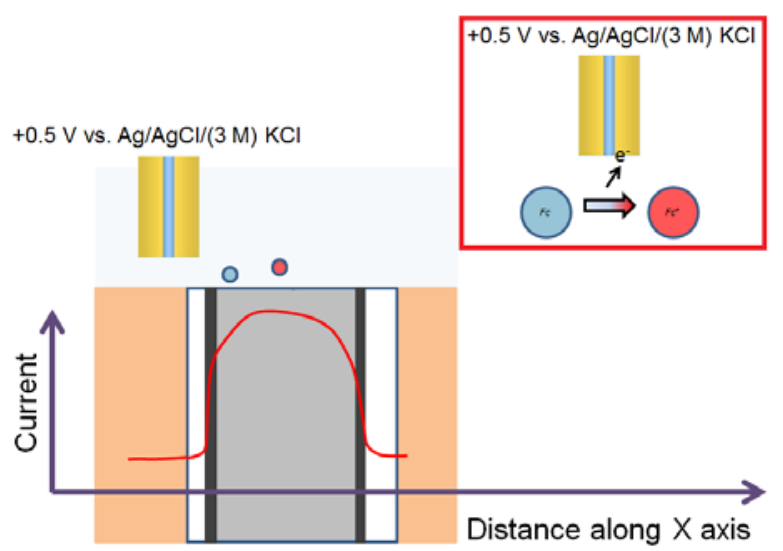

Figure 3 - Sketch describing the faradaic current measured at the platinum tip for ferrocenemethanol oxidation as the tip travels above the cut edge.

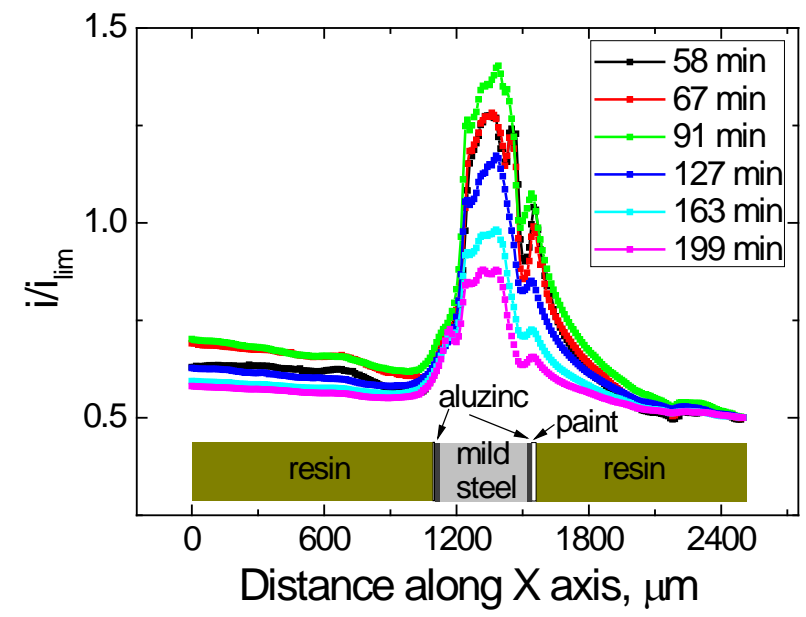

(A)
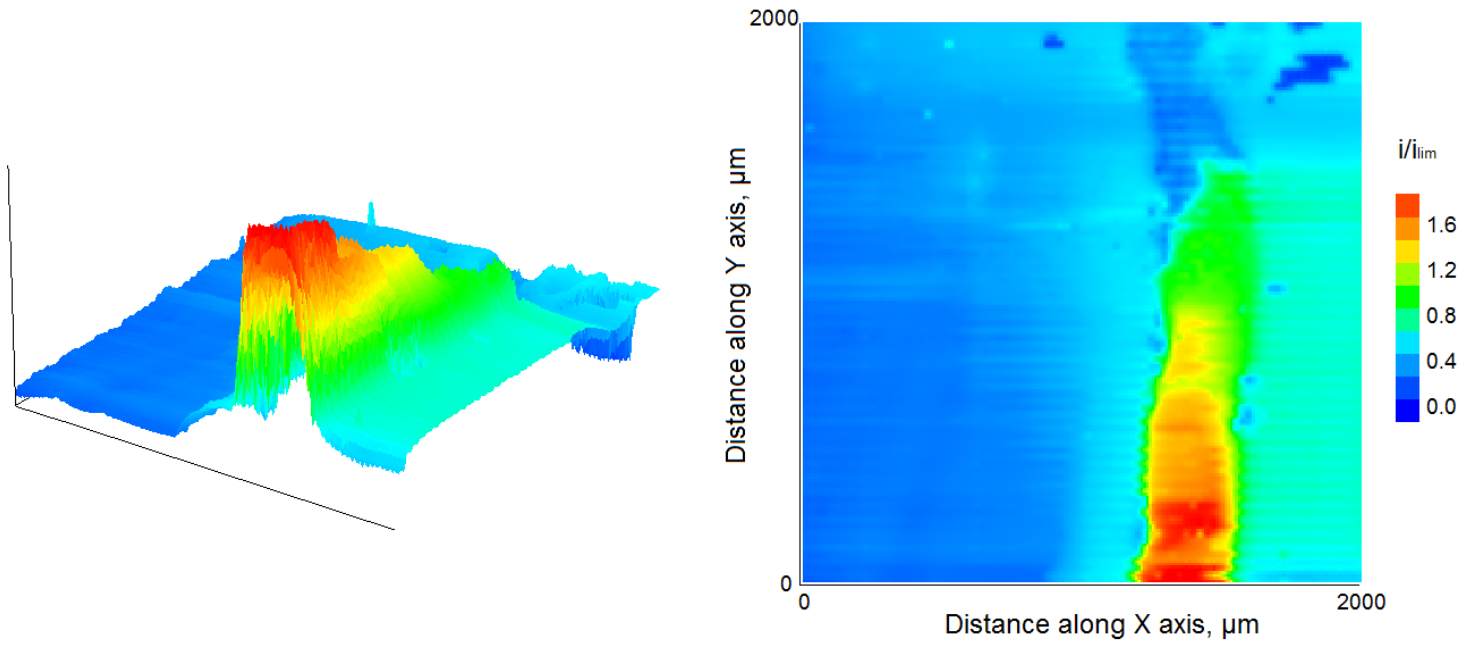

(B)

Figure 4 - (A) scan lines at different immersion times indicated in the graph, and (B) SECM image after 24 minutes immersion in $1 \mathrm{mM} \mathrm{NaCl}$ of electrochemical activity towards regeneration of ferrocene-methanol over the cut edge; the time required to record this image was 103 minutes. $E_{t i p}=+0.50 \mathrm{~V}$ vs. $\mathrm{Ag} / \mathrm{AgCl} /(3 \mathrm{M} \mathrm{KCl})$; tip-substrate distance: $15 \mu \mathrm{m}$. Scan rate: $7 \mu \mathrm{m} / \mathrm{s}$. 


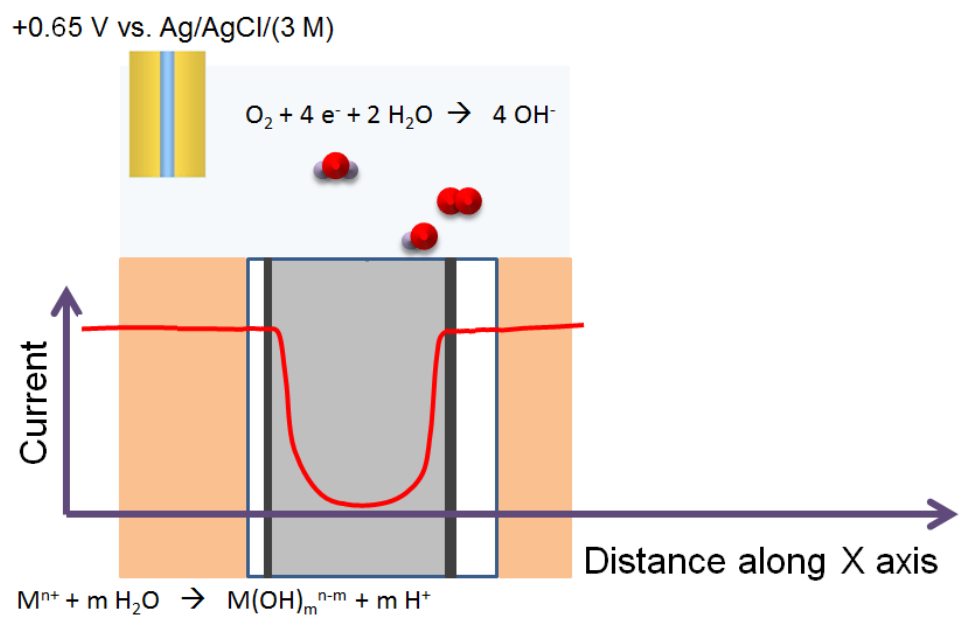

Figure 5 - Sketch describing the faradaic current measured at the platinum tip for the reduction of naturally dissolved oxygen in the electrolyte as the tip travels above the cut edge.

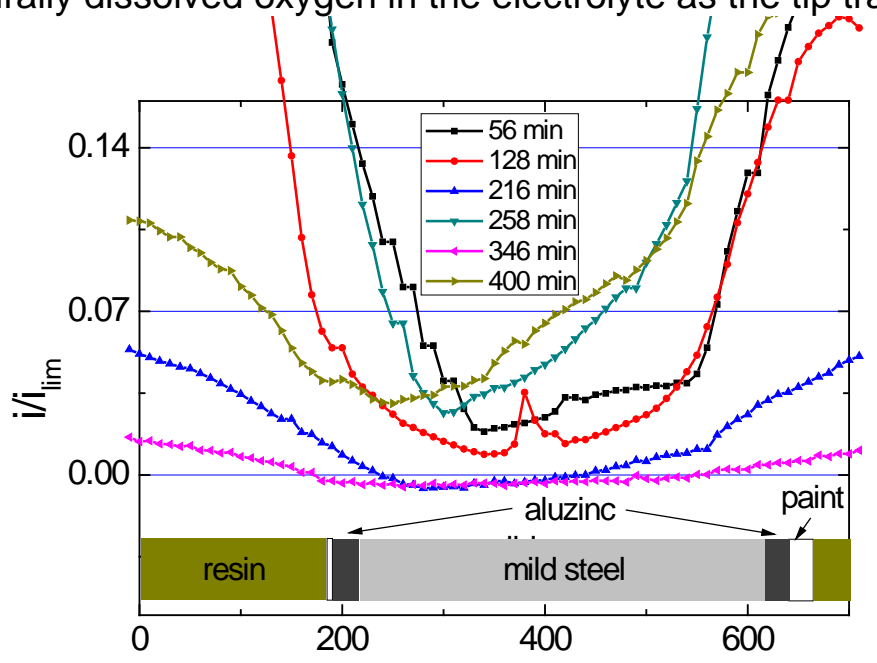

Distance along $X$ axis, $\mu \mathrm{m}$

Figure 6 - Distribution of oxygen concentration above the cut edge in $1 \mathrm{mM} \mathrm{NaCl}$ for immersion times indicated in the graph. $E_{t i p}=-0.65 \mathrm{~V}$ vs. $\mathrm{Ag} / \mathrm{AgCl} /(3 \mathrm{M}) \mathrm{KCl}$; tip-substrate distance: $15 \mu \mathrm{m}$. Scan rate: $7 \mu \mathrm{m} / \mathrm{s}$.

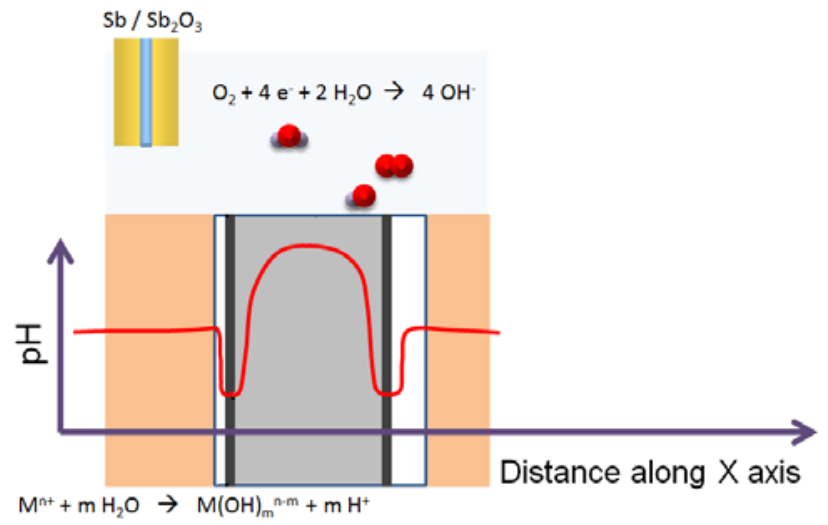

Figure 7 - Sketch describing the $\mathrm{pH}$ changes occurring at the cut edge as result of the reduction of oxygen on steel (cathodic sites, local alkalization), and combined dissolution and hydrolysis of zinc and aluminium from the aluzinc layers (anodic sites, local acidification). 


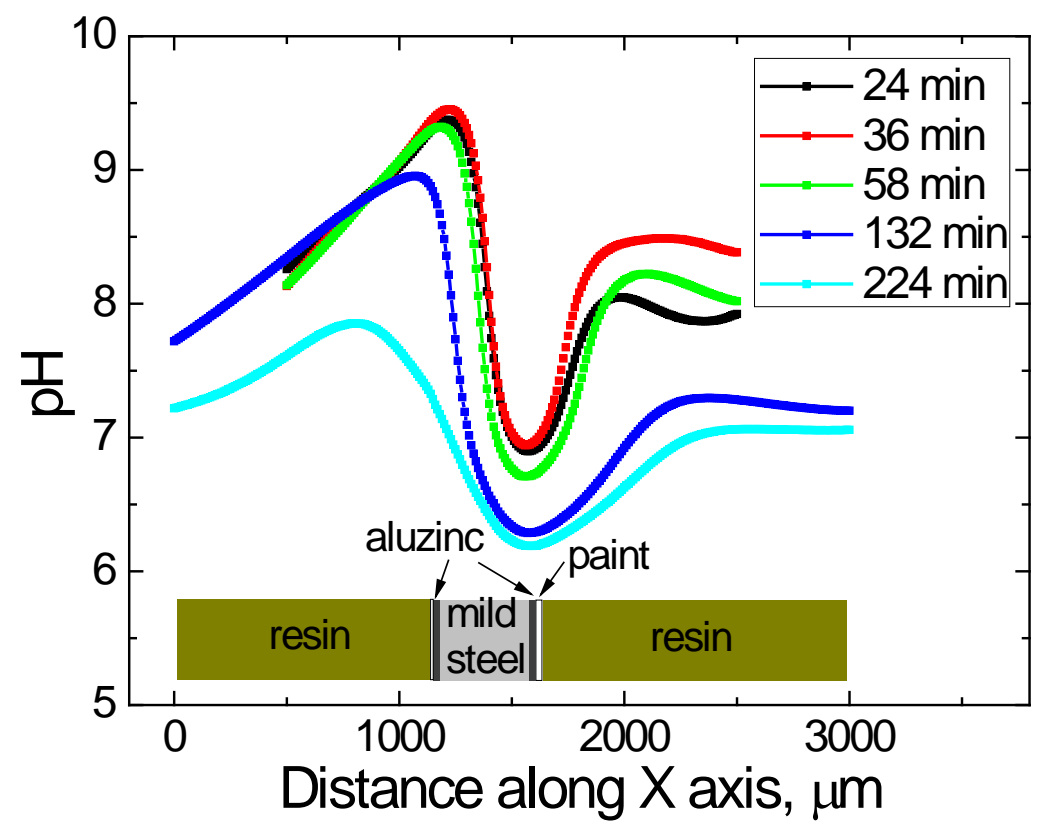

(A)

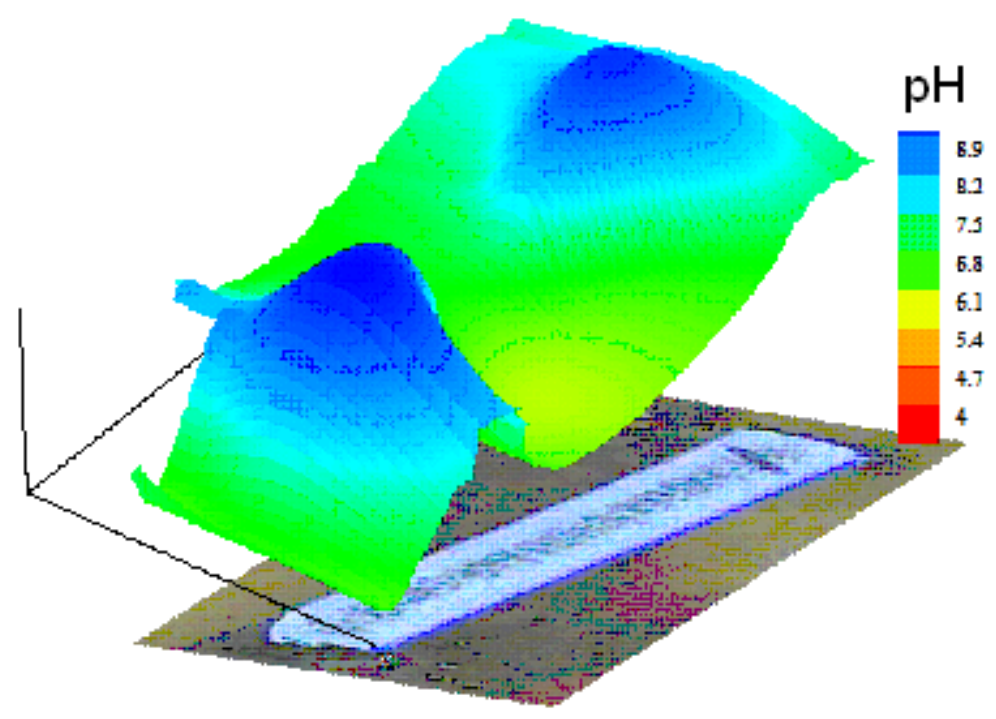

(B)

Figure 8 - $\mathrm{pH}$ distribution in: $(\mathrm{A})$ a line over the cut edge for different immersion times as indicated in the graph, and (B) a plane parallel to the surface of the cut edge after immersion in $1 \mathrm{mM} \mathrm{NaCl}$ for $5 \mathrm{~h}$; the time required to record this image was 210 minutes. Tip-substrate distance: $15 \mu \mathrm{m}$. Scan rate: $7 \mu \mathrm{m} / \mathrm{s}$. 